Theatre Research in Canada

Recherches théâtrales au Canada

NEVEUX, OLIVIER, Contre le théâtre politique. Paris : La

fabrique éditions, 2019, $320 \mathrm{pp}$.

\title{
Camille Gascon
}

Volume 41, numéro 2, 2020

URI : https://id.erudit.org/iderudit/1074804ar

DOI : https://doi.org/10.3138/tric.41.2.r03

Aller au sommaire du numéro

Éditeur(s)

University of Toronto Press

ISSN

1196-1198 (imprimé)

1913-9101 (numérique)

Découvrir la revue

Citer ce compte rendu

Gascon, C. (2020). Compte rendu de [NEVEUX, OLIVIER, Contre le théâtre

politique. Paris : La fabrique éditions, 2019, 320 pp.] Theatre Research in Canada

/ Recherches théâtrales au Canada, 41(2), 324-326.

https://doi.org/10.3138/tric.41.2.r03 d'utilisation que vous pouvez consulter en ligne.

https://apropos.erudit.org/fr/usagers/politique-dutilisation/ 


\title{
NEVEUX, OLIVIER \\ Contre le théâtre politique. \\ Paris : La fabrique éditions, 2019, 320 pp.
}

\author{
CAMILLE GASCON \\ Université de Montréal, Montréal, Québec, Canada.
}

Alors que se multiplient sur les scènes occidentales des pratiques artistiques dites " citoyennes ", Olivier Neveux approfondit sa réflexion sur le théâtre et ses engagements à travers un nouvel essai au titre provocateur, Contre le théâtre politique. Dans la foulée de son ouvrage précédent, Politiques du spectateur. Les enjeux du théâtre politique aujourd'hui (2013), l'auteur adopte une perspective critique peu commune à l'heure actuelle dans le champ de la recherche théâtrale, influencée notamment par la pensée marxiste. Ainsi, contrairement à ce qu'il laisse entendre par l'intitulé de son ouvrage, Neveux ne rejette pas l'art théâtral politique (au contraire), mais problématise une alliance qui semble aujourd'hui aller de soi. Accordés l'un à l'autre, conciliés dans leurs besoins respectifs, théâtre et politique ne peuvent plus se déstabiliser et, de ce fait, se neutralisent mutuellement. Convoquant, pour nourrir sa réflexion, celle de théoriciens du théâtre (Erwin Piscator et Bertolt Brecht notamment) et de philosophes (en particulier Jacques Rancière, mais aussi Daniel Bensaïd), Neveux propose de désunir les deux termes afin de leur redonner leur sens propre. Il fait le pari que, de cet écart, pourrait surgir une rencontre inédite entre les intelligences respectives du théâtre et de la politique, sur des bases moins évidentes, une rencontre qui ne soit non plus collaborative, mais, au contraire, tumultueuse, agressive et, de ce fait, plus fructueuse.

Pour ce faire, l'auteur déboulonne un mythe tenace du théâtre qui entretient l'idée que celui-ci est, par essence, politique, mais qui, du même coup, évince la politique du théâtre. Car "l'inflation de sa nomination" (I2) cache une perte, la possibilité que la politique offre, lorsqu'elle n'est pas " rationaliste ", d'ouvrir " la pensée à l'hypothèse des alternatives » et de fragiliser "l'évidence de l'existant» (233). Si les formes scéniques actuelles la dévitalisent, force est de constater, comme le démontre Neveux dans le premier chapitre, «La dé-politique culturelle ", qu'elles s'accordent en fait trop bien aux "politiques culturelles " et à leurs présupposés. Les gouvernements français qui se sont succédé (mais nous ne pouvons qu'être frappés par les parallèles possibles avec le Québec), de François Hollande à la "start-up nation » d'Emmanuel Macron (26), achèvent de faire de l'art une marchandise prise dans la logique économique néolibérale et qui doit répondre de son "utilité ", du " profit social évaluable " qu'il génère (66). Le théâtre, pour justifier son existence, se fait pédagogue et travailleur social. Il a le devoir de contribuer à un vivre-ensemble pacifié pour pallier les "manques " (d'empathie, de savoir, de liens), les fractures sociales provoquées par une politique devenue "science des résolutions gestionnaires " (28). L'œuvre est réduite à ce qu'elle est censée produire, affecter, alors qu'en retour la politique dans l'art se thématise et se formate. Olivier Neveux lève le voile des évidences pour montrer ce qu'il y a de politique dans l'alliance entre le théâtre et la politique. 
Après avoir dressé un état de la politique théâtrale, l'auteur tente de cerner, dans le chapitre « Du trop de réalisme », les formes qu'empruntent les pratiques scéniques actuelles explicitement politiques, voire polémiques. Il s'interroge sur certains tropes de ce théâtre, notamment l'écrasante domination de la réalité (qui en serait devenue «le maître mot » [I73]) et l'injonction à la représenter (considérée comme d'emblée critique). Lauteur rappelle ainsi que, dans Ça ira (I) - Fin de Louis de Joël Pommerat (pièce qui a connu un succès public et critique), l'apparente neutralité du point de vue (revendiquée aussi par bon nombre de créateurs de théâtre documentaire) n'a pour effet que de reconduire une vision coutumière de la politique, sans remise en question de la légitimité des pouvoirs. Si l'œuvre est tenue de représenter la réalité, elle doit aussi avoir un effet émancipateur supposé qui se traduit par un recours très fréquent aux témoignages et à la participation du public (le théâtre doit libérer la parole, "activer » le spectateur). Neveux, de nouveau, refuse de s'en tenir à l'évidence : « comment peut-on vouloir programmer l'émancipation de l'autre, sauf à le maintenir sous sa tutelle [...] ? " (I64).

Enfin, après avoir questionné la fabrique de la politique au sein de certaines créations, l'auteur propose, dans le dernier chapitre de l'ouvrage, "Lart du théâtre ", des pistes de réflexion sur ce que pourrait celui-ci pour la politique. Il cerne deux de ses points fondateurs qui participent de son "intelligence ", de sa façon de faire spécifique, et qui lui permettent de travailler la politique : le fictif et le manque. Considérer le théâtre comme un art qui « fait avec le manque " (242) et " travaille au fictif " (248) entraîne un déplacement de l'attention de la réalité vers la représentation. Cette perspective, à contrecourant de la doxa qui associe "théâtre politique » et interprétation du monde, a pour effet de faire intervenir le théâtre sur un terrain qui lui est propre, celui des représentations. Neveux suggère que ce serait possiblement en s'interrogeant sur les représentations dans lesquelles nous sommes immergées, et en premier lieu celles qui régulent notre vision du théâtre, de la politique et de leur alliance, que cet art trouverait sa plus féconde inspiration politique.

Contre l'« évidente » association du théâtre et de la politique, Neveux propose des points de rencontre plus subtils, des passages souterrains où ils peuvent se rencontrer pour « conspirer " (275). S'il pave la voie à certaines réflexions pour ceux qui « s'échinent à mobiliser le théâtre contre ce monde » (I7), l'auteur évite, malgré ce que le titre laisse présager, de tenir un discours de domination qui ferait jouer une pensée du théâtre contre une autre. Il s'écarte de toute tentative programmatique, ne cherchant pas à « modéliser » une pratique artistique, mais plutôt à multiplier les significations, à remettre la pensée en mouvement. En guise de conclusion, il propose une analyse de Deux Mille Dix Sept de Maguy Marin (20I7), un spectacle frontalement politique, qui, plutôt que d'exemplifier ses propos précédents, l'oblige à « s'embarrasser à son tour et rappeler à toutes tentatives généralisantes, y compris les siennes, qu'elles butent, heureusement, sur la singularité des œuvres [...]» (289).

Si le regard d'Olivier Neveux, dans cet essai d'une actualité indéniable, est avant tout orienté vers les scènes européennes, force est de constater que la problématique qu'il soulève se pose aussi devant le théâtre québécois. La politique s'y retrouve sous les mêmes formes attendues et les mêmes injonctions pèsent sur lui. Il n'y a qu'à consulter les orientations du Conseil des arts du Canada pour le réaliser : l'art doit contribuer à « contrer la fragmentation et la marginalisation [...], stimuler l'engagement civique [...] et générer un sentiment renouvelé d'appartenance » (Brault). Son appui financier se justifie ainsi par la fonction sociale qu'il est sommé de remplir (il faut Faire cuvre utile, pour reprendre le titre d'une émission diffusée 
sur la chaîne ICI ARTV et les artistes sont tenus de multiplier les activités de médiation culturelle). De plus, la désaffection du politique à l'égard de la culture déplorée par Neveux est ici aussi observable (alors que le bénévolat et la paupérisation demeurent la norme). On ne peut que souhaiter que cet essai pertinent puisse contribuer à ce que le théâtre, au Québec comme en Europe, libéré de l'obligation de représenter le monde, redevienne cette zone autonome «tramée de fictif»(272).

\section{Bibliographie}

Brault, Simon. Léquité dans les arts : ouvrir les portes pour un avenir meilleur, discours du directeur et chef du Conseil des arts du Canada, Halifax, Prismatic Arts Festival, 2I septembre 2016.

Marin, Maguy. Deux Mille Dix Sept, Centre Culturel André Malraux, Vandoeuvre-Lès-Nancy, 5-8 octobre 2017 .

Neveux, Olivier. Politiques du spectateur. Les enjeux du théâtre politique aujourd'hui. La Découverte, 2013.

Pommerat, Joël. Ça ira (I) - Fin de Louis, Centre national des Arts, I6-19 mars 2016.

\section{Contributrice}

CAMILLE GASCON est doctorante au département des littératures de langue française de l'Université de Montréal sous la direction de Jean-Marc Larrue et de Claire Legendre. Dans le cadre de sa recherche, soutenue par le CRSH (Bourse d'études supérieures du Canada JosephArmand-Bombardier), elle s'intéresse à la construction esthétique de l'authenticité dans les théâtres documentaires. Son essai «Véro ou le nouveau gynécée " paraitra prochainement dans l'ouvrage collectif Mythologies québécoises. 